\title{
Balkanologie
}

Balkanologie Revue d'études pluridisciplinaires

Vol. X, $n^{\circ} 1-2 \mid 2008$

Volume $X$ Numéro 1-2

\section{La crise caucasienne à travers le premier journal bulgare de Constantinople, le Carigradski vestnik,} 1854-1859

Pierre Voillery

\section{OpenEdition}

Journals

Édition électronique

URL : http://journals.openedition.org/balkanologie/362

DOI : 10.4000/balkanologie.362

ISSN : 1965-0582

Éditeur

Association française d'études sur les Balkans (Afebalk)

Référence électronique

Pierre Voillery, «La crise caucasienne à travers le premier journal bulgare de Constantinople, le Carigradski vestnik, 1854-1859 », Balkanologie [En ligne], Vol. X, n 1-2 | 2008, mis en ligne le 03 juin 2008, consulté le 17 décembre 2020. URL : http://journals.openedition.org/balkanologie/362 ; DOI https://doi.org/10.4000/balkanologie.362

Ce document a été généré automatiquement le 17 décembre 2020.

(c) Tous droits réservés 


\title{
La crise caucasienne à travers le premier journal bulgare de Constantinople, le Carigradski vestnik, 1854-1859
}

\author{
Pierre Voillery
}

1 A première vue, la présence dans le premier journal bulgare de Constantinople de mentions éparses des révoltes caucasiennes de la moitié du XIX ${ }^{\circ}$ siècle ne semble guère mériter que quelques lignes, l'idée principale étant que l'opposition des Caucasiens à l'annexion russe n'a que peu retenu l'attention des Bulgares de Constantinople. En y regardant de plus près, le sujet prend une autre dimension. Chamyl fut, dans les années 1840-1850, une coqueluche des milieux libéraux occidentaux, avant que l'enlèvement de princesses géorgiennes en 1854 ne lui aliène ces sympathies et que la fin de la guerre de Crimée ne lui retire son utilité stratégique pour les Occidentaux ${ }^{1}$. Les Circassiens deviennent, avec les Tchetchènes, le peuple qui peut contribuer à faire reculer le rouleau compresseur russe. La lutte contre l'expansionnisme russe dans le Caucase est une priorité de la Couronne britannique dont les agents parcourent la région, où se déroule le Grand Jeu. Le Portfolio de David Urqhart, écossais, agent secret, publiciste et zélateur du droit des peuples à combattre la Russie, est à la pointe de ce combat ${ }^{2}$.

2 Au milieu du XIX ${ }^{\circ}$ siècle, en Occident, le Caucase préoccupe et Chamyl passionne. En 1848 sort à Francfort sur le Main un ouvrage aujourd'hui encore considéré comme la référence sur la question dans le domaine militaire comme le souligne l'Encyclopédie de l'Islam³. En 1854 , le mélodrame chanté que lui consacre P. Maurice à Paris connaît un succès certain ${ }^{4}$. Le même succès s'observe outre Manche avec, en 1856, la publication par L. Moser de The Caucasus and its people 5 . En 1859, Alexandre Dumas contribue à populariser sa cause à la suite de son Voyage au Caucase ${ }^{6}$. Dès lors, il paraît justifié de se pencher sur le traitement de la question caucasienne par le Carigradski vestnik, journal autour duquel se structure l'identité bulgare entre 1850 et 1862 mais aussi passeur d'idées occidentales et plus particulièrement françaises, et de tenter de démêler ce qui 
est strictement bulgare de ce qui peut être interprété comme une influence extérieure, ottomane, occidentale ou russe.

3 En bonne méthode, la première opération est l'identification du corpus disponible. Si le dépouillement fut long en raison du nombre de pages (environ 2700 pages) dans lequel il fallut chercher, il ne fut pas pour autant difficile et le corpus réuni au bout du compte est plutôt maigre. L'hypothèse initiale paraissait ainsi se confirmer : le sujet n'avait pas véritablement concerné les Bulgares de Constantinople. Pourtant, la seule mention de cette révolte dans des pages si précieuses pour la cause bulgare et la formation politique de ce peuple montrait que, a contrario, les élites de la capitale s'y étaient bien intéressées. Dès lors, le sujet méritait d'être examiné de plus près.

4 L'étroitesse du corpus indiquait la marche à suivre. Il était inutile d'espérer révéler des aspects inattendus des révoltes de Chamyl, de ses nabi, de ses mudir, d'un côté et des Circassiens de l'autre, qui on fait l'objet d'une littérature plus souvent militante et hagiographique que scientifique ${ }^{7}$. Il était tout aussi illusoire de vouloir prouver que ces révoltes ont pu, ne fut-ce qu'à un moment, figurer au nombre des préoccupations des Bulgares du temps. A l'inverse, la lecture des maigres éléments disponibles montre que la manière dont le sujet avait été traité dans ce journal, sa fréquence, le contenu des articles et l'origine de l'information jettent une lumière intéressante sur la presse ottomane de l'époque d'une part, et que, d'autre part, Tchétchènes et Circassiens ne bénéficient pas d'un même regard.

\section{Le Carigradski Vestnik}

Il faut d'abord s'interroger sur ce que représente le Carigradski Vetsnik? Quelles sont ses options ? Quel rôle joue-t-il parmi les Bulgares ? En quoi popularise-t-il cette cause hors des limites naturelles de cette communauté en phase de structuration identitaire et nationale? En quoi fut-il un passeur d'idées exogènes, et plus particulièrement occidentales parmi les Bulgares?

6 Fondé en 1848, il ne connaît son véritable essor qu'à partir de 1850, après avoir été repris par Alexandre Exarh qui l'anime jusqu'à sa fermeture en 1862. Personnage central de la construction de l'identité culturelle et nationale bulgare, il naît en 1810 et meurt en 1891. Ce fils de notables bulgares de Stara Zagora aura été boursier à Paris où il se lie avec tous les grands noms du tanzimat. Revenu à Constantinople après avoir obtenu l'appui de la Russie, il crée ou aide plus de trente écoles, donne un nouvel essor à la revendication bulgare pour une église séparée du Patriarcat orthodoxe avant de passer treize années à l'ambassade ottomane à Paris aux termes desquelles il devient le premier préfet chrétien de Plovdiv en 1879 et sera à deux reprises candidat au trône de Bulgarie 8 .

7 Le journal occupe une place unique dans l'histoire bulgare'. Il n'est pas le premier à être publié et ne sera pas le dernier. Mais, entre 1848 et 1858, il est l'unique journal bulgare de l'Empire et ne sera ensuite jamais véritablement concurrencé par les autres publications, qui, soit n'ont qu'une existence éphémère ou épisodique, soit représentent des intérêts étrangers aux Bulgares, comme le catholique Bălgarija de Dragan Cankov qui sort à partir de 1858. Il présente une position éditoriale originale, refusant d'être partisan sauf lorsqu'il s'agit de la défense, de l'illustration et de la promotion des intérêts bulgares. Ouvert à tous, il s'intéresse à tout et sait s'adapter aux circonstances. Ce pragmatisme fait qu'il ne sera censuré qu'une seule fois dans un 
contexte où le contrôle préalable était manifestement absent du secteur de la presse et le pouvoir peu désireux de s'immiscer dans les conflits entre le Patriarcat et ses ouailles.

8 Il porte dignement le drapeau bulgare dans un panorama médiatique ottoman limité ${ }^{10}$. Dans ses Lettres sur la Turquie, Ubicini le mentionne (en se trompant toutefois sur son nom puisqu'il parle de Novina bulgarska), parmi les rares journaux publiés dans L'Empire ottoman ${ }^{11}$. Certes, son tirage, quatre cent exemplaires hebdomadaires, le place loin derrière le Moniteur ou des deux journaux francophones de la capitale, le Journal de Constantinople et l'Echo Orient. Mais il est l'unique journal minoritaire non confessionnel en ce sens que, s'il défend une identité orthodoxe des Bulgares, il ne fait pas de la dimension religieuse son seul axe de bataille, bien au contraire. Et sa seule existence, dans un tel paysage, lui confère un poids particulier.

9 Le Carigradski Vetsnik est sorti régulièrement chaque samedi. Par sa diffusion et sa couverture de l'actualité bulgare à Constantinople et surtout dans les provinces, il touche l'ensemble des communautés. Pour la première fois, celles-ci sont réunies dans un même réseau, autour d'une même source d'information et d'un support d'idées dans lequel elles se reconnaissent d'autant plus aisément que son propriétaire leur laisse généreusement la parole. De ce point de vue, entre autres, ce journal est sans nul doute le premier élément collectif et fédérateur de l'identité bulgare, l'école ne constituant pas un réseau centralisé mais la résultante d'initiatives individuelles ou communautaires, l'Eglise ne devenant un facteur d'unification qu'après que son indépendance eut été déclarée unilatéralement en 1860.

10 C'est également un passeur d'idées étrangères et modernes ainsi que de nouvelles du monde. Si les questions bulgares occupent la majeure partie de ses quatre feuilles, l'actualité nationale et étrangère y joue un rôle très important, permettant d'informer le lecteur de ce qui se passe dans l'Empire et à l'extérieur, comme d'introduire des concepts politiques modernes comme l'a montré Vera Boneva ${ }^{12}$. Il reprend les articles publiés par d'autres, traitant plus particulièrement l'actualité française et l'ascension de Napoléon III ainsi que la guerre de Crimée. D'autres sujets sont également régulièrement évoqués dans ses colonnes ${ }^{13}$. C'est dans ce contexte que s'inscrit la publication de brèves sur la conquête russe du Caucase et la révolte de Chamyl.

\section{Corpus}

Le dépouillement systématique du journal sur la totalité de son existence aboutit à un total de 40 occurrences.

Sur ces quarante occurrences, six portent sur des points étrangers à la question caucasienne (chemin de fer, épidémies, inondations). Restent donc trente quatre occurrences relatives à Chamyl, aux Circassiens et aux opérations russes dans le Caucase.

13 S'il fallait procéder à une évaluation quantitative de l'importance attribuée par le journal à cette question, un critère pourrait être la surface occupée par cette question par rapport à la surface totale publiée par le journal exprimée en espace/caractère. Le résultat est révélateur. La superficie, exprimée en nombre de caractères, consacrée à la question caucasienne s'élève à 47278 signes. Chaque numéro du journal comporte l'équivalent espaces/caractères de 12 colonnes de 82 lignes à 44 caractères soit 43300 
caractères par numéro. Soit, pour 676 numéros représente 29268000 caractères. La surface imprimée occupée par la question caucasienne est alors de $0,15 \%$ ou bien l'équivalent d'un numéro sur $676 \ldots$

\section{Chronologie}

14 C'est la guerre de Crimée avec ses extensions caucasiennes qui fait entrer Chamyl et la Circassie dans ses colonnes.

15 En 1854, quatre articles sont consacrés aux opérations militaires dans le Caucase, dans lesquels Chamyl et les Circassiens apparaissent directement. Dans un cas, c'est son gendre qui est le héros de l'action, dans deux cas, c'est lui-même qui est impliqué, dans un dernier cas, c'est un membre de sa parentèle. Le sujet en est à chaque fois la volonté de résistance de montagnards tcherkesses, qu'ils mettent les Russes en difficulté, qu'ils se fédèrent devant la menace russe, qu'ils bénéficient de l'aide de la France contre la Russie.

Rien d'étonnant à ce que la guerre de Crimée soit le décor de l'émergence de Chamyl et de la Circassie sur la scène de la presse bulgare stambouliote. Plusieurs raisons l'expliquent. La première est qu'elle implique l'Empire ottoman, dont les Bulgares, comme les autres populations minoritaires, tiennent à réaffirmer leur fidélité alors que les troupes sont engagées. Dans le cas bulgare, l'intérêt est accentué par le fait que la Russie est l'ennemie, alors qu'elle est vue comme la sœur orthodoxe. Le conflit à un effet direct sur la ligne suivie par le journal qui, jusqu'en 1856, met sous le boisseau ses revendications contre le haut clergé. Simultanément, les opérations militaires et les tractations politiques sont suivies avec le plus grand soin par les Bulgares qui sont ainsi parfaitement informés de la situation ${ }^{14}$. Enfin, les Puissances alliées de l'Empire ottoman, n'hésitent pas à appuyer Chamyl contre l'ennemi du moment, le Russe ${ }^{15}$.

En 1855, le journal publie deux articles sur l'insurrection kurde, l'afflux de réfugiés circassiens dans l'Empire et l'importance des pertes russes.

1856 est encore une année quantitativement maigre avec seulement quatre occurrences. La guerre de Crimée est finie. Le Traité de Paris conclu. Pourtant, c'est cette année là que l'on trouve deux articles importants puisque celui du 29 septembre mentionne l'offensive victorieuse des rebelles contre les Russes et surtout leur refus d'accepter les résultats du traité de Paris selon lesquels la Circassie est rattachée à la Russie. Le second article (8 décembre) mentionne l'importance des forces rebelles placés sous le commandement de trois chefs : Chamyl, Sefer et Ismail.

19 Le suivi devient dès lors plus précis. En 1857, le journal publie dix-sept articles, soit un toutes les trois semaines en moyenne pour un total de 15602 signes. La presse russe est de plus en plus fréquemment citée. La prééminence de Chamyl s'affirme par la répétition de son nom. Si la rébellion se renforce à la lecture du journal, la volonté russe d'en finir également avec le renforcement des troupes et surtout l'envoi d'artillerie depuis la Pologne. La Russie marque des points mais connaît également des défaites dont les lecteurs bulgares sont informés comme ils le sont des tractations entre la Grande Bretagne, la Perse et la Russie.

20 Avec onze occurrences, l'année 1858 reste importante même si elle marque le début de la décrue caucasienne. Les nouvelles sont de plus en plus contradictoires puisque, à lire les dépêches provenant de Russie, Chamyl passe son temps à se subir des défaites et à se 
rendre, alors que les nouvelles d'origine différente ne cesse de parler de la résistance du rebelle et des revers qu'il inflige aux Russes.

1859 est la dernière année de référence, avec seulement trois articles en septembre sur la « reddition » de Chamyl dont le lecteur comprend, trois semaines plus tard, qu'il est certes acculé dans une place refuge mais encore libre...

Les deux dernières années, 1860 et 1861, sont marquées par la publication d'informations sur des épidémies dans le Caucase et sur une forme de toux dénommée « toux caucasienne »...

\section{Provenance de l'information}

23 Le contenu de l'information, et donc le message véhiculé volontairement ou non, est étroitement corrélé aux sources de presse qu'il reproduit fidèlement et donc à leurs pays d'origine. En effet, ni Alexandre Exarh, ni ses rédacteurs successifs ne prennent la plume sur le sujet. C'est n'est pas leur priorité. Ils se contentent de reprise de journaux étrangers. C'est tout à la fois un choix contraint, faute d'autres sources, et délibéré, par la préférence donnée à telle source plutôt qu'à une autre?

24 C'est à partir de la presse ottomane, occidentale ou russe que le Carigradski Vestnik alimente ses colonnes. Sur les trente sept sources mentionnées, vingt trois sont explicitement mentionnées comme étant des journaux. Il est cependant probable que les sources dont l'origine n'est pas précisée («on écrit de Russie," «lettre de Trébizonde » par exemple ») sont également journalistiques.

La provenance géographique de ces journaux montre que si la Russie est absente les deux premières années, elle apparait comme fournisseur d'information en 1856 avec deux références. Elle domine en 1857 avec onze mentions sur 16 et reste majoritaire en 1857 avec six références sur 13. Au total, elle compte pour vingt des trente sept occurrences. Deux revues sont plus fréquemment citées: l'Invalide et Caucase. Les informations provenant de Saint Petersbourg, de Tiflis ou de Russie sont très probablement extraites de journaux, parfois mentionnées comme «le journal russe » de 1857 ou le « journal russe publié à Cracovie » publié la même année. Le ton général en est assez propagandiste. Il s'agit là d'information officielle, voire de propagande.

Trébizonde, donc des sources non russes, vient ensuite avec huit occurrences également réparties entre 1854 et 1858. Il est impossible de déterminer par quels canaux ces nouvelles parviennent depuis les rives de la mer noire au journal bulgare. Comme il n'a pas de correspondants, il doit reprendre ces dépêches de journaux qu'il ne cite pas mais qui sont incontestablement favorable au parti ottoman et qui sont en outre assez précises dans leur contenu. La presse constantinopolitaine francophone (Le Journal de Constantinople et La Presse d'Orient) compte sept occurrences entre 1854 et 1859, les journaux occidentaux pour cinq, dont la Gazette de Cologne, La Patrie de Paris, le Lloydd de Trieste et des journaux européens globalement cités et « un certain journal allemand ». Leur seule lecture montre un ton sensiblement plus libre que celui de la presse russe. C'est de l'information contrôlée.

27 Information contrôlée plutôt que propagande ? Contrairement à ce que laisse entendre Nuri Inuğur dans son article Naissance et développement de la presse dans l'Empire ottoman ${ }^{16}$, le pouvoir ottoman n'est guère tatillon envers la presse. Un exemple suffit à le démontrer. En quinze années, le Carigradski vestnik qui mène de dures attaques contre 
le haut clergé grec, n'a fait l'objet de censure qu'une fois, et encore d'une portée limitée puisqu'il ne s'agit pas d'une interdiction de publication. La mesure est également réduite dans son effet puisque les attaques reprennent de plus belle un mois après ${ }^{17}$. Le ton général de la presse demeure très libre et c'est dans ce contexte que s'inscrit la politique de reprise d'articles par le journal bulgare comme le ton des articles repris. En ce sens, on peut parler d'information contrôlée, si ce n'est auto-contrôlée plutôt que surveillée.

Une étude plus précise des sources étrangères du Carigradski Vestnik montre que son équipe rédactionnelle dispose d'un très large éventail de journaux de référence à partir desquels il alimente son lectorat en nouvelles étrangères ${ }^{18}$. C'est dans ce cadre que s'inscrit sa politique de communication sur la crise. La comparaison permet de mettre en évidence un élargissement de son vivier sur ce point précis puisque ni les deux journaux russes, ni le Lloyd de Trieste ou La Patrie de Paris ne figurent au nombre des références usuelles du journal.

Si l'on comprend par quels canaux certaines informations sont parvenues au journal bulgare, d'autres questions demeurent à ce stade sans réponse. Comment les informations dont l'origine nous est inconnue sont-elles parvenues entre les mains de la rédaction du Carigradski Vestnik? De quels journaux s'agit-il, si l'hypothèse qu'il s'agit bien de journaux, se confirme? Faut-il voir, au moins pour les nouvelles russes et comme c'est probable, un approvisionnement par l'ambassade russe à Constantinople plutôt qu'une volonté du journal d'élargir ses sources? A l'appui de cette idée, Il faut relever que l'élargissement à la presse russe intervient en 1856, soit au moment où la phase militaire, et donc aiguë, de la guerre de Crimée, se termine et laisse place à phase de tractations diplomatiques, plus propice à la reprise d'articles de la presse réputée d'un Etat « ennemi de l'Empire ottoman » comme à l'action de propagande du palais de Russie auprès des médias du Bosphore. Autre question : comment interpréter la rupture que l'on observe entre novembre 1858 et septembre 1859, période pendant laquelle le journal ne donne aucune information sur la question? Faut-il y voir une adhésion du journal à la distance prise par la presse constantinopolitaine francophone devant le caractère contradictoire des informations qu'il a diffusées en 1858 dans $\mathrm{N}^{\circ} 342$ ? L'année de silence qui suit incite à le penser.

\section{Russie, Caucase, Circassie, Tchétchénie et Chamyl. Le Carigradski Vestnik et la crise caucasienne}

Existe-t-il une ligne du journal vis à vis de la question caucasienne? Autrement dit, le journal fait-il le choix d'une cause plutôt qu'une autre ? La tentation est grande de dire que le journal aurait soutenu le parti russe puisqu'il était bulgare, de le soupçonner de parti pris anti-russe puisqu'il défendait des options pro-occidentales, de rechercher enfin dans ses colonnes une identité d'intérêts, ou, à tout le moins des aspirations communes avec la lutte d'un autre peuple.

31 La lecture des dépêches montre que, si ces préoccupations ne lui sont pas totalement étrangères, c'est avec discrétion qu'elles s'expriment et qu'elles ne sont perceptibles que dans le cadre d'un examen général du journal, ce que les lecteurs, qui l'achetaient chaque semaine pour connaître l'actualité bulgare avant tout, ne pouvaient mesurer. La question du Caucase appartient à l'actualité factuelle et c'est sous cet angle qu'elle est 
avant tout perçue. Le journal est, dans cette affaire mineure à ses yeux, pris entre son désir de ne pas se mettre en porte-à-faux vis à vis des autorités ottomanes, pour lesquelles la révolte de Chamyl devient dès 1856 une cause de préoccupation alors qu'elles sortent d'une pénible guerre de Crimée avec la Russie, son aspiration au progrès que représente malgré tout la Russie face à ces guerriers incontrôlés et musulmans, son désir de modernité que défend le journal et qui se traduit par un véritable attrait pour ce qui se passe en France et une absence d'intérêt pour l'actualité russe. De même évoque-t-il le cas des Circassiens de manière aussi discrète quoi qu'en termes plus positifs.

S'ajoute à cela une incapacité à examiner les sources d'un œil critique, faute de pouvoir en vérifier le contenu, donc la véracité. Le N 342 du 17 août 1857 est l'un des deux moments où la rédaction laisse percer son scepticisme sur les informations qu'elle diffuse et une certaine distance vis à vis de la presse russe. Il écrit que si l'Invalide russe annonce un succès russe, des correspondances de Trébizonde parlent d'un succès de Chamyl et que, dans le doute, le Journal de Constantinople préfère se référer aux journaux européens qui font, cependant, référence à l'Invalide. L'autre moment où le doute aurait pu se lever est la reddition de Chamyl, annoncée triomphalement par la presse russe reprise par le journal bulgare $\left(\mathrm{N}^{\circ} 447\right)$, pour être tempérée une semaine après (De Saint Petersbourg, "Chamyl s'est rendu »; d'Erzerum, «Chamyl se serait rendu») et contredite deux semaines plus tard ( $\mathrm{N}^{\circ} 450$, «Chamyl s'est enfermé dans Gunib avec plusieurs centaines de murids »). Enfin, l'annonce, prématurée, de la reddition de Chamyl permet au journal, qui reprend la Presse d'Orient, de se lancer dans un article admiratif sur cet homme qui « a fait comme Abd El Kader ». Encore la France.

Quel est le message géopolitique qui se dégage des titres et informations ainsi diffusés? La lecture du récent ouvrage de Eric Hoesli ${ }^{19}$ rappelle utilement que, dans le Grand Jeu ainsi que le nommait les Britanniques, le Caucase comprenait au moins deux acteurs en lutte contre la pénétration russe et objets des convoitises anglaises: les « montagnards » de Chamyl et les Circassiens. Entre les deux, le traitement réservé par le journal n'est pas le même.

Bien que limitée et sans que n'y apparaisse une quelconque adhésion, une sympathie pour la cause circassienne se manifeste à deux reprises. La première est la mention de l'envoi par le chef rebelle Sefer Pacha d'un émissaire pour rappeler aux Russes, traité ou pas $\left(\mathrm{N}^{\circ} 296\right)$, que la Circassie est indépendante. Ce faisant, le journal relaie de fait l'information selon laquelle si les opérations russes sont juridiquement légitimes, elles se heurtent à une opposition politique de cette population qui proclame son droit à l'indépendance. Le second moment pendant lequel le journal donne la parole aux Circassiens est la publication dans le $\mathrm{N}^{\circ} 360$ à partir du Lloyd de Trieste, d'un mémoire aux Puissances du Grand Conseil circassien en date du 9 octobre 1857 dans lequel il exprime, dans des termes qui fleurent bon son diplomate britannique, l'espoir de voir reconnaître les droits de son peuple par le Traité de Paris.

L'examen des rubriques dans lesquelles le sujet est évoqué incite à la même conclusion $^{20}$. Si, au début, la révolte de Chamyl a son existence éditoriale autonome, très rapidement, à partir de 1856, elle est mentionnée sous le chapeau éditorial «Russie ». L'année 1857 est encore marquée par une brève hésitation en janvier, mais dès le mois de juin, le pli est pris. La révolte de Chamyl et de ses "montagnards" s'inscrit clairement dans un contexte territorial russe. A l'inverse, les Circassiens figurent soit sous le chapeau " Russie », soit sous la rubrique « Circassie » sans mention 
de la Russie. L'impression se dégage qu'ils ont un statut plus indépendant, et donc plus empreint de noblesse, que les partisans de Chamyl selon la rédaction du journal dont la révolte, une fois terminée la guerre de Crimée, relève du fait divers, certes héroïque, mais non du droit des peuples. A l'inverse, si le droit des Circassiens n'est pas affirmé en tant que tel, les rédacteurs éprouvent une certaine difficulté à juger leur révolte illégitime puisqu'ils ne jugent manifestement pas légitime leur subordination à la Russie au point d'en faire l'intitulé des rubriques.

Bien plus que l'affaiblissement de l'intérêt stratégique pour les guerre du Caucase, qui ne justifie en rien une telle différence de traitement, c'est sans doute sérieux déficit d'image dont est victime Chamyl depuis l'enlèvement des princesses géorgiennes début juillet 1854. Cet événement a été largement couvert par la presse occidentale en raison, entre autres, de la présence d'une Française ${ }^{21}$ parmi les victimes de cette longue séquestration. Certes, les Circassiens sont aussi cruels, aussi violents, aussi impitoyables mais ils ont des «porte-paroles » dont la voix se fait entendre dans certains milieux occidentaux. La lecture des arguments incite à y voir la marque d'habitués des chancelleries et plus particulièrement celle des agents britanniques. Mais cela, les hommes du Carigradski vetsnik l'ignorent. Les Circassiens n'ont pas non plus commis l'imprudence de s'en prendre à des princesses innocentes accompagnées d'une dame de compagnie française sachant écrire... Et en outre, le fait n'est pas négligeable, ils sont connus dans l'Empire ottoman comme dans l'Occident qui en rêve (qu'on relise les visites aux marchés aux esclaves de presque tous les voyages en Orient), pour la beauté de leurs femmes qui peuplent les harems et qui donnèrent au Trône impérial nombre de ses occupants. Plus que Chamyl qui prend une coloration de bandit d'honneur, ou d'un de ces rebelles primitifs étudiés par E. Hobsbawn ${ }^{22}$, ce sont les Circassiens qui, à en lire le Carigradski Vestnik, pourraient prétendre au titre de représentants caucasien du Printemps des Nations.

37 La première conclusion que l'on peut tirer de la couverture de la crise caucasienne par le Carigradski Vestnik est, sans surprise, que les Bulgares de la capitale, au milieu du XIX ${ }^{\circ}$ siècle, sont avant tout préoccupés par leur propre devenir et ont les yeux tournés vers les pays occidentaux, plus particulièrement la France ou l'Angleterre. Leur attitude vis à vis de la Russie est plus complexe. A leurs yeux, c'est un pays slave qui attire autant par sa capacité d'intervention militaire, et donc de rupture de la domination ottomane, qu'un Etat dont la politique autoritaire intérieure ne séduit personne, et donc les manœuvres parfois brutales et souvent ambiguës vis à vis des Slaves ottomans orthodoxes suscite une réelle méfiance.

La seconde conclusion porte sur la corrélation étroite entre l'information diffusée et son origine. En effet, dans le Carigradki Vestnik, journal bulgare s'il en est, ce ne sont jamais les Bulgares qui parlent du Caucase. Ce sont des informations reprises directement et sans modification provenant d'autres journaux que diffuse le journal. Il ne dispose pas de correspondant, encore moins d'envoyé. C'est sans aucune distance, ni capacité d'appréciation qu'il reproduit les dépêches ou les articles d'autres journaux. Jamais il n'émet d'opinion qui lui soit propre. Quand il s'agit de reprises de la presse russe, les rebelles sont présentés défavorablement et les opérations russes héroïsées. A l'inverse, dans les journaux occidentaux ou constantinopolitains, le jugement est plus modéré et le caractère propagandiste moins apparent, mais non négligeable comme le traitement du cas circassien le montre. 

d'abord y voir l'écho de l'intérêt qu'elles suscitent dans la presse occidentale, qui exerce une influence indéniable sur un homme, Alexandre Exarh, profondément attaché à la France et à la culture politique française. La classe politique française s'intéressait à Chamyl. Les Britanniques au révolté tchétchène et aux Circassiens. La presse francophone de Constantinople s'y intéressait donc. La presse britannique aussi. La presse russe également. Rien d'étonnant à ce notre Bulgare en parle. Pour autant, il ne se prononce jamais sur la question elle-même, diffusant le plus souvent, par la simple reprise d'articles, la position officielle selon laquelle le Caucase est attribué à la Russie en vertu du traité de Paris. Et c'est avec une prudence dont il est difficile de dire si le lecteur en percevait le sens, qu'il témoigne de sa préférence envers les Circassiens face à la révolte de Chamyl.

voyons enfin une élite intéressée par une actualité située au-delà de son horizon et soucieuse d'informer ses lecteurs sur des évènements lointains. En agissant ainsi, le Carigradski vestnik contribue à former la culture géo-politique d'une nation en train de naître comme il l'a fait en abordant bien d'autres sujets, à première vue bien éloignés de la question bulgare ${ }^{23}$. Il eut été étonnant qu'un journal bulgare se préoccupât de la question caucasienne davantage que de la construction de l'identité bulgare. Ce qui doit nous étonner est bien qu'il s'y soit intéressé et qu'il en ait reflété les différentes sensibilités. Preuve que, dès sa naissance la presse communautaire ottomane, et plus particulièrement bulgare, a eu une vision large et contemporaine de sa mission, dont il n'est pas certain qu'elle l'a conservée tout au long de son histoire...

\section{NOTES}

1. Art. Shāmil, Encyclopédie de l'Islam, Nouvelle édition, Tome IX, Leiden 1996, pp. 292-296.

2. Hoesli (Eric), La conquête du Caucase, Paris 2006. Ouvrage très bien documenté et récent.

3. Bodenstedt (F.), Die Völker des Kaukasus und ihre Freiheitskämpfe gegen die Russen. Ein Betrag zur neuesten Geschichte des Orients, Frankfurt am Main, 1848.

4. Le titre en est «Chamyl » et l'histoire très éloignée de la réalité. Cité par E. Hoesli, op. cit, p. 95

5. Moser (L.), The Caucasus and its people, with a brief history of their wars and a sketch of the achievements of the renowned chief Schamyl.Londres 1856

6. Dumas (A.), Voyage au Caucase, également publié sous le titre Le Caucase (Paris 1859). Ce très long ouvrage, conforme au style de Dumas (64 chapitres), est le récit du séjour qu'il fit dans la région avec les troupes russes. Dans le chapitre XXVIII (Chamyl, ses femmes, ses enfants), il fait une description pleine de noblesse et de respect du personnage.

7. Parmi les ouvrages les plus récents: M. Gammer, Muslim resistance to the Tsar: Shamil and the conquest of Chechnia and Daghestan, Londres- New York 1992. Très récent, signe d'un intérêt renouvelé lié à l'actualité : Branch (L.), Les sabres du paradis, Paris 2004.

8. Voillery (P.), Alexandre Exarh. Un destin bulgare, à paraître, Ed. Colibri, Sofia. En bulgare.

9. Voillery (P.), «Contribution à l'histoire de la presse bulgare ottomane. Le premier journal bulgare d'Istanbul. Le Carigradski Vestnik. 1848-1862 », Meždu dva svjata, Bălgarite v Rumelja. Entre 
deux mondes, les Bulgares de Roumélie, Sofia 2005. En bulgare. Sous presse en français dans Identité et nationalité chez les Bulgares ottomans. Alexandre Exarh (1810-1891), Istanbul, Isis press.

10. Clayer (N.), Popovic (A.), Zarcone (T.), Presse turque et presse de Turquie, Istanbul-Paris 1992. Lorsque le Carigradski Vestnik commence de paraître, la presse ottomane n'est plus embryonnaire mais le panorama qu'elle offre n'en est pas moins limité. Le Ceride-i Havadis, le journal officiel de l'Empire, également publié en arabe, grec, persan, arménien est français, tire entre 100000 et 150000 exemplaires. Le journal officiel du khédive d'Egypte, le Vekkayi-i Misriyye, autour de 30 000. La presse francophone est très présente. Elle est dominée par Moniteur ottoman, le premier journal officiel de l'Empire. Il faut également mentionner l'influent Journal de Constantinople, Echo de l'Orient qui sera racheté, en 1858, par le propriétaire de l'Impartial, journal de Smyrne, fondé en 1841 et de tendance anglophile. A cela s'ajoutent plusieurs titres liés à des intérêts communautaires, qu'ils soient grecs, arméniens ou juifs.

11. Ubicini (A.), Lettres sur l'Empire ottoman, Paris,1851. Lettre VII, p. 182.

12. Boneva (Vera), «La nouvelle constitution française. Les institutions constitutionnelles modernes des pays européens dans les pages du Carigradski Vestnik (1850-1860) » In Vălčev (G.), Voillery (P.), Alexandre Exarh et les routes bulgares vers l'Europe. Colloque international de Stara Zagora, 8/9 mai 2005. Edition bilingue français-bulgare. A paraître.

13. Bur-Markovska (M.), «Die bulgarischen Zeitung Carigradski Vestnik (Konstantinopler Zeitung) über die Ereignisse in Ungarn 1848-1849 », Bulgarian Historical Review, 1995,2, pp. 12-42.

14. La comparaison des fréquences statistiques des articles consacrées à ces différents sujets ne laisse aucun doute quant à la perception très politique que le propriétaire du journal a du contexte et de sa marge d'action. Voillery (P.), «Contribution à l'histoire de la presse bulgare ottomane... » Art. cit.

15. Les livres sur le sujet mettent l'accent sur l'appui britannique. Pourtant, le Carigradski vestnik le 11 septembre 1854 mentionne la fourniture par la France de 12000 fusils à Chamyl. Chiffre considérable, événement important passé sous silence qui montre, s'il est avéré, que, au moins pendant la guerre de Crimée, Paris pensa au Caucase.

16. Presse turque... op. cit. pp. 83-92

17. Voillery (P.), «Contribution à l'histoire de la presse bulgare ottomane... » Art. cit.

18. Voillery (P.), «Contribution à l'histoire de la presse bulgare ottomane... » Art. cit. Les titres les plus fréquemment cités dans le Carigradski Vestnik sont les suivants: France : La Semaine Française, Le Moniteur, Le Journal des Débats, Le Sémaphore de Marseille, l'Opinion Publique, Le Constitutionnel, le Vrai Journal, Le Siècle. Empire Ottoman: Le Journal de Constantinople, le Courrier d'Orient, L'Impartial, Le Journal de Smyrne, Amalthia (Smyrne), Byzantion, Bălgarija. Allemagne: Gazette d'Augsbourg, Gazette de Prusse, Gazette de Cologne, Journal de Cassel. Grande Bretagne: Times, Standard, Globe, Morning advertiser. Belgique: Le Siècle. Grèce: Amalthia. Italie: Corriere Italiano, Journal de Naples. Autriche: Observateur de Trieste, Courrier Mercantile (Trieste). Divers: Portfolio (Malte), Ispania (Espagne), Journal de Porto, Journal de Lisbonne

19. La conquête du Caucase, op. cit.

20. Si la mention géographique du «Caucase » est fréquente, de temps à autres apparaissent des références aux régions qui le composent et aux populations qui l'habitent. C'est incontestablement là le fruit de l'érudition ethnographique accumulée et par les Russes et par les Britanniques. Quelques noms aujourd'hui connus des seuls spécialistes de la région apparaissent comme la Mingrélie, le Daghestan, l'Aikratka. D'autres ont connu une fortune contemporaine comme l'Abkhasie, le bas et le haut Karabak, la Thétchénie. Des noms de peuples enfin tels que les Pataki ou les Skapani.

21. Drancey (A.), Captive des Tchétchènes, Paris 2006. Le livre a été initialement publié en 1856 par son cousin, Merlieux (E.), sous le titre Souvenirs d'une française captive de Chamyl.

22. Hobsbawn (E.), Les primitifs de la révolte dans l'Europe moderne, Paris, Fayard, 1966.

23. Boneva (Vera), « La nouvelle constitution française.. », art. cit. 


\section{RÉSUMÉS}

Carigradski Vetsnik, fondé en 1848, est le seul journal bulgare publié au sein de l'Empire ottoman entre 1848 et 1858. Quelles sont ses options? Quel rôle joue-t-il parmi les Bulgares? En quoi popularise-t-il la crise caucasienne hors des limites naturelles de la communauté bulgare en phase de structuration identitaire et nationale? En quoi fut-il un passeur d'idées exogènes, et plus particulièrement occidentales parmi les Bulgares?

\section{INDEX}

Index géographique : Caucase, Istanbul, Russie, Empire ottoman, Circassie

Mots-clés : Question caucasienne, Presse bulgare, Chamyl 